\title{
GROUND-WATER QUALITY IN THE CACUAS-JUNCOS VALLEY, PUERTO RICO, APRIL TO OCTOBER 1990
}

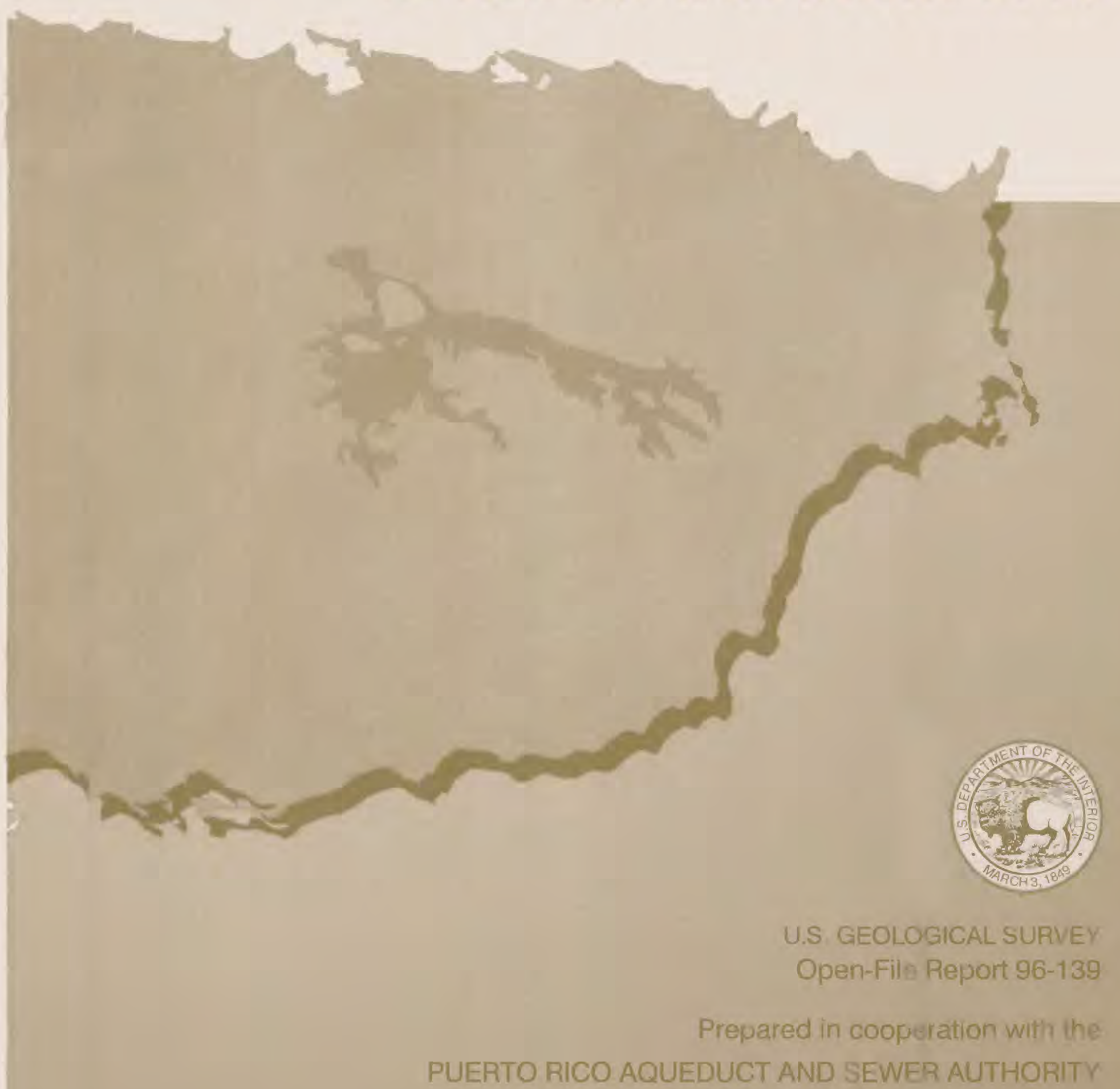


Water samples from 19 wells were collected and analyzed for trace metals (table 3 ). Although barium was present in all samples, concentrations were far below the primary maximum contaminant level (MCL) $(2,000 \mu \mathrm{g} / \mathrm{L})$ and the Puerto Rico MCL (PRMCL) (1,000 mg/L) for drinking water (table 5). Water from wells located near the urban center of Juncos $(32,35,36$, and 41 through 44) had the highest concentrations of barium (140 to $290 \mu \mathrm{g} / \mathrm{L}$ ). Cadmium was detected in samples from public-supply wells 19 and 36, and from wells 32, 41, and 46. However, cadmium concentrations were below the PRMCL $(10 \mu \mathrm{g} / \mathrm{L})$ and the maximum permissible concentration $(5 \mathrm{mg} / \mathrm{L})$ for a potable water source. Lead was detected in only 3 of 19 samples and all lead concentrations were below the PRMCL (50 $\mu \mathrm{g} / \mathrm{L})$ and the maximum permissible concentration $(50 \mu \mathrm{g} / \mathrm{L})$. Molybdenum was detected only in water from well 46 (an agricultural well); the molybdenum concentration of that sample was only $10 \mu \mathrm{g} / \mathrm{L}$, the lower detection limit. Silver was detected in samples from four wells and the spring; all silver concentrations were far below the Federal and Commonwealth MCL's. Strontium was detected in all 19 samples. Vanadium was detected in six samples. Zinc was detected in 18 samples; all zinc concentrations were far below the SMCL for drinking water. Lithium, in low concentrations, was detected in 10 of 19 samples.

Volatile synthetic organic chemicals (VOC), widely used in industrial processes, were detected in water from wells 5, 9, 15, 29, and 46 (table 4). Chloroform was detected in water from four of these wells in concentrations ranging from 0.4 to
$2.5 \mu \mathrm{g} / \mathrm{L}$. Dichlorodifluoromethane (Freon 12) was detected in well 46 at a concentration of 3.6 $\mu \mathrm{g} / \mathrm{L}$. Concentrations of dichloroethylene and trichloroethylene ( 0.4 and $0.7 \mu \mathrm{g} / \mathrm{L}$, respectively), below the drinking-water MCL, were detected in samples from one industrial well (well 5). Concentrations of benzene $(5,800 \mu \mathrm{g} / \mathrm{L})$, ethylbenzene $(1,200 \mu \mathrm{g} / \mathrm{L})$, toluene $(12,000$ $\mu \mathrm{g} / \mathrm{L})$, and xylene $(15,000 \mu \mathrm{g} / \mathrm{L})$, all of which exceeded the MCL, were detected in samples from well 9. VOC were not detected in samples collected from public-water supply wells.

The laboratory results were checked for accuracy using the cation-anion balance and the specific conductance-dissolved solids ratio analyses. In the cation-anion balance analysis, the sum of cations expressed in milliequivalents per liter should equal the sum of anions expressed in the same units. The difference between the sum of the cations and the sum of the anions should be less than 5 percent of the total anions and cations present. The sample from well 5 was the only one that exceeded the 5 percent limit.

The specific conductance-dissolved solids ratio for water of ordinary composition should be between 0.55 and 0.75 (Hem, 1989). All the samples had ratios within that range, except for the samples from well 10 and site 4 , which had higher ratios. The higher ratio in these two sites may be caused by the high concentration of sulfate. Water with high sulfate concentrations may reach or even exceed the upper end $(0.75)$ of the specific conductance-dissolved solids ratio (Hem, 1989). 
Table 1. Information and ground-water field measurements from selected wells and a spring within the Caguas-Juncos alluvial valley, east-central Puerto Rico

$\left[\mu \mathrm{S} / \mathrm{cm}\right.$, microsiemens per centimeter at $25^{\circ} \mathrm{C} ;{ }^{\circ} \mathrm{C}$, degrees Celsius; mg/L, milligrams per liter; $\mu \mathrm{g} / \mathrm{L}$, micrograms per liter; nd. not detected;

-- , information not available; na, not applicable]

\begin{tabular}{|c|c|c|c|c|c|c|c|c|c|}
\hline $\begin{array}{l}\text { Well } \\
\text { number }\end{array}$ & Well name & $\begin{array}{c}\text { Well identification } \\
\text { number }\end{array}$ & Use of water & $\begin{array}{c}\text { Well } \\
\text { depth } \\
\text { (in feet } \\
\text { below } \\
\text { land } \\
\text { surface) }\end{array}$ & $\begin{array}{c}\text { Screened } \\
\text { interval } \\
\text { (in feet } \\
\text { below } \\
\text { land } \\
\text { surface) }\end{array}$ & $\begin{array}{c}\text { Date of } \\
\text { sampling }\end{array}$ & $\begin{array}{c}\text { Specific } \\
\text { conduct- } \\
\text { ance } \\
(\mu \mathrm{s} / \mathrm{cm})\end{array}$ & $\begin{array}{c}\mathrm{pH} \\
\text { (units) }\end{array}$ & $\begin{array}{l}\text { Tem- } \\
\text { perature } \\
\text { (C) }\end{array}$ \\
\hline 1 & Pozo Viejo AAA & 181538066021300 & public & 116 & $79-100$ & $08-27-90$ & 485 & 7.4 & 26.5 \\
\hline 2 & CJ-TW 15 & 181539066014500 & observation & 70 & $25-70$ & $08-08-90$ & 554 & 7.0 & 26.0 \\
\hline 3 & Caguas Sugar & 181511066005700 & industrial & -- & -- & $06-27-90$ & 712 & 7.0 & 26.5 \\
\hline 4 & Caguitas Spring & 181459066013600 & none & na & na & $06-29-90$ & 2.350 & 7.3 & 31.0 \\
\hline 5 & Quality Elect. & 181424066025400 & industrial & 81 & $51-81$ & $09-10-90$ & 3,480 & 7.0 & 27.0 \\
\hline 6 & CJ-TW 20 & 181446066013400 & observation & 37 & $25-35$ & $07-11-90$ & 924 & 7.9 & 29.5 \\
\hline 7 & CJ-TW 1 & 181445066011500 & observation & 32 & $26-30$ & $08-07-90$ & 3.200 & 6.6 & 29.0 \\
\hline 8 & Cartagena 2 & 181480066034500 & agricultural & 270 & -- & $08-30-90$ & 959 & 6.8 & 26.5 \\
\hline 9 & CJ-TW2 & 181422066015300 & observation & 45 & $39-43$ & $09-11-90$ & - & - & - \\
\hline 10 & CJ-TW 22 & 181412066020700 & observation & 52 & $25-50$ & $08-02-90$ & 468 & 6.7 & 27.0 \\
\hline 11 & CJ-TW 7 & 181406066004600 & observation & 76 & $51-71$ & $08-09-90$ & 934 & 7.0 & 28.0 \\
\hline 12 & CJ-TW 13 & 181351066013900 & observation & 73 & $20-70$ & $08-06-90$ & 444 & 6.5 & 27.0 \\
\hline 13 & CJ-TW 19A & 181352066025300 & observation & 67 & $50-65$ & $07-19-90$ & 512 & 6.5 & 28.0 \\
\hline 14 & Gravero Navarro & 181337066004100 & industrial & - & - & $04-19-90$ & 1.020 & 7.6 & 27.0 \\
\hline 15 & R. Diez & 181332066022100 & commercial & 80 & -- & $07-30-90$ & 322 & 6.3 & 28.5 \\
\hline 16 & CJ-TW 12 & 181318066032600 & observation & 70 & $45-65$ & $08-08-90$ & 368 & 6.4 & 30.0 \\
\hline 17 & CJ-TW 11 & 181311066022500 & observation & 110 & $66-96$ & $07-23-90$ & 253 & 6.9 & 28.0 \\
\hline 18 & E. Delgado & 181046066024700 & agricultural & - & -- & $08-02-90$ & 471 & 7.7 & 26.0 \\
\hline 19 & Gurabo AAA 6 & 181548065592900 & public & 123 & $60-123$ & $08-20-90$ & 1,100 & 7.1 & 26.0 \\
\hline 20 & Gurabo AAA 7 & 181549065592100 & public & 160 & $54-136$ & $05-01-90$ & 586 & 7.1 & 26.0 \\
\hline 21 & CJ-TW 17 & 181511065592200 & observation & 45 & $20-40$ & $07-10-90$ & 536 & 7.4 & 28.0 \\
\hline 22 & CJ-TW 21A & 181602065584400 & observation & 65 & $30-60$ & $07-12-90$ & 427 & 8.0 & 27.0 \\
\hline 23 & O. Dávila & 181544065583200 & domestic & -- & -- & $04-26-90$ & 942 & 7.2 & 27.0 \\
\hline 24 & CJ-TW 18 & 181540065580300 & observation & 65 & $40-60$ & $07-18-90$ & 577 & 7.2 & 27.0 \\
\hline 25 & P. Morales & 181524065573900 & industrial & 40 & -- & $08-23-90$ & 769 & 7.0 & 27.0 \\
\hline
\end{tabular}


Table 1. Information and ground-water field measurements from selected wells and a spring within the Caguas-Juncos alluvial valley, east-central Puerto Rico--Continued

\begin{tabular}{|c|c|c|c|c|c|c|c|c|c|}
\hline $\begin{array}{l}\text { Well } \\
\text { number }\end{array}$ & Well name & $\begin{array}{c}\text { Well identification } \\
\text { number }\end{array}$ & Use of water & $\begin{array}{l}\text { Well } \\
\text { depth } \\
\text { (in feet } \\
\text { below } \\
\text { land } \\
\text { surface) }\end{array}$ & $\begin{array}{c}\text { Screened } \\
\text { interval } \\
\text { (in feet } \\
\text { below } \\
\text { land } \\
\text { surface) }\end{array}$ & $\begin{array}{c}\text { Date of } \\
\text { sampling }\end{array}$ & $\begin{array}{c}\text { Specific } \\
\text { conduct- } \\
\text { ance } \\
(\mu \mathrm{s} / \mathrm{cm})\end{array}$ & $\underset{\text { (units) }}{\mathrm{pH}}$ & $\begin{array}{c}\text { Tem- } \\
\text { perature } \\
\text { (C) }\end{array}$ \\
\hline 26 & El Bambu AAA & 181622065571500 & public & 300 & $40-300$ & $08-22-90$ & 585 & 7.4 & 27.0 \\
\hline 27 & Berríos 1 & 181504065571700 & domestic & - & - & $04-30-90$ & 941 & 7.4 & 26.5 \\
\hline 28 & Don Sico AAA & 181618065565900 & public & 300 & $40-300$ & $08-22-90$ & 884 & 7.4 & 26.0 \\
\hline 29 & C. Sánchez & 181503065565600 & agricultural & 110 & - & $07-09-90$ & 1,180 & 7.8 & 27.0 \\
\hline 30 & E. Mendoza 1 & 181518065561400 & agricultural & 110 & - & $06-28-90$ & 450 & 7.2 & 26.0 \\
\hline 31 & CJ-TW $3 \mathrm{~A}$ & 181513065554600 & observation & 87 & $62-82$ & $07-12-90$ & 351 & 7.4 & 27.5 \\
\hline 32 & CJ-TW 4A & 181501065555500 & observation & 34 & $23-33$ & $07-16-90$ & 299 & 6.3 & 28.0 \\
\hline 33 & CJ-TW 4B & 181501065555501 & observation & 144 & $119-139$ & $07-16-90$ & 416 & 7.7 & 28.0 \\
\hline 34 & Z. Méndez 2 & 181514065661100 & agricultural & 250 & - & $08-01-90$ & 715 & 7.5 & 27.0 \\
\hline 35 & Juncos AAA 1 & 181437065552200 & public & 140 & $40-140$ & $04-23-90$ & 447 & 6.7 & 26.5 \\
\hline 36 & Juncos AAA 2 & 181437065551900 & public & 320 & $120-320$ & $07-31-90$ & 760 & 6.8 & 27.0 \\
\hline 37 & Juncos AAA 7 & 181432065552000 & public & 90 & $50-90$ & $07-31-90$ & 377 & 6.9 & 26.5 \\
\hline 38 & Juncos AAA 5 & 181424065551400 & public & 85 & $27-85$ & $04-23-90$ & 617 & 7.0 & 26.5 \\
\hline 39 & Juncos AAA 3 & 181432065550500 & public & 88 & $40-88$ & $07-30-90$ & 647 & 7.0 & 27.0 \\
\hline 40 & Finca Batey & 181453065545400 & agricultural & -- & -- & $08-27-90$ & 374 & 6.9 & 27.0 \\
\hline 41 & CJ-TW 16A & 181448065544200 & observation & 95 & $70-90$ & $07-10-90$ & 242 & 7.4 & 26.0 \\
\hline 42 & CJ-TW 16B & 181448065544200 & observation & 60 & $30-50$ & $07-17-90$ & 220 & 6.3 & 26.5 \\
\hline 43 & CJ-TW 6 & 181415065545400 & observation & 102 & $67-87$ & $08-06-90$ & 1,130 & 7.5 & 27.5 \\
\hline 44 & CJ-TW 23 & 181330065540000 & observation & 50 & $34-49$ & $07-17-90$ & 739 & 6.9 & 28.0 \\
\hline 45 & E. Berríos 2 & 181402065523200 & agricultural & -- & - & $08-23-90$ & 469 & 7.0 & 27.0 \\
\hline 46 & Santa Ana 1 & 181315065523100 & agricultural & 120 & -- & $04-30-90$ & 761 & 7.2 & 26.0 \\
\hline 47 & Hermosura 2 & 181206065514400 & agricultural & - & -- & $07-26-90$ & 483 & 6.6 & 28.0 \\
\hline 48 & Robles Mendoza & 181345065503200 & agricultural & - & -- & $07-26-90$ & 165 & 7.7 & 27.5 \\
\hline 49 & Hacienda Cuco 2 & 181220065502300 & agricultural & -- & -- & $04-26-90$ & 841 & 7.1 & 27.0 \\
\hline
\end{tabular}


Table 2. Major ions and nutrients in ground water at selected wells and a spring within the Caguas-Juncos alluvial valley, east-central Puerto Rico

[mg/L, milligrams per liter; $\mu \mathrm{g} / \mathrm{L}$, micrograms per liter; --, data not available; $<$, less than the detection limit]

\begin{tabular}{|c|c|c|c|c|c|c|c|c|c|}
\hline $\begin{array}{l}\text { Well } \\
\text { number }\end{array}$ & Well name & $\begin{array}{c}\text { Date of } \\
\text { sampling }\end{array}$ & $\begin{array}{c}\text { Calcium, } \\
\text { dissolved } \\
(\mathrm{mg} / \mathrm{L})\end{array}$ & $\begin{array}{c}\text { Magnesium, } \\
\text { dissolved } \\
(\mathrm{mg} / \mathrm{L})\end{array}$ & $\begin{array}{c}\text { Sodium, } \\
\text { dissolved } \\
(\mathrm{mg} / \mathrm{L})\end{array}$ & $\begin{array}{l}\text { Sulfate, } \\
\text { dissolved } \\
\text { (mg/L) }\end{array}$ & $\begin{array}{l}\text { Chloride, } \\
\text { dissolved } \\
(\mathrm{mg} / \mathrm{L})\end{array}$ & $\begin{array}{c}\text { Flouoride, } \\
\text { dissolved } \\
(\mathrm{mg} / \mathrm{L})\end{array}$ & $\begin{array}{c}\text { Silica, } \\
\text { dissolved } \\
\text { (mg/L as } \\
\mathrm{SiO}_{2}\end{array}$ \\
\hline 1 & Pozo Viejo AAA & $08-27-90$ & 47 & 18 & 24 & 25 & 28 & 1.2 & 44 \\
\hline 2 & CJ-TW 15 & $08-08-90$ & 52 & 21 & 28 & 14 & 44 & .1 & 50 \\
\hline 3 & Caguas Sugar & $06-27-90$ & 65 & 15 & 61 & 83 & 51 & $<.1$ & 37 \\
\hline 4 & Caguitas Spring & $09-11-90$ & 420 & 5 & 200 & 1,100 & 180 & .1 & 24 \\
\hline 5 & Quality Elect. & $09-10-90$ & 48 & 36 & 31 & 70 & 53 & 1.4 & 68 \\
\hline 6 & CJ-TW 20 & $07-11-90$ & 40 & 11 & 140 & 100 & 99 & .3 & 69 \\
\hline 7 & CJ-TW 1 & $08-07-90$ & 480 & 58 & 280 & 1,600 & 74 & .4 & 54 \\
\hline 8 & Cartagena 2 & $08-30-90$ & 88 & 51 & 36 & 92 & 78 & .1 & 69 \\
\hline 10 & CJ-TW 22 & $08-02-90$ & 47 & 19 & 20 & 30 & 29 & .7 & 49 \\
\hline 11 & CJ-TW 7 & $08-09-90$ & 100 & 12 & 84 & 69 & 23 & .3 & 33 \\
\hline 12 & CJ-TW 13 & $08-06-90$ & 28 & 15 & 36 & 17 & 37 & .4 & 21 \\
\hline 13 & CJ-TW 19A & $07-19-90$ & 30 & 17 & 47 & 25 & 53 & .1 & 77 \\
\hline 14 & Gravero Navarro & $04-19-90$ & 130 & 1 & 90 & 240 & 73 & .2 & 32 \\
\hline 15 & R. Diez & $07-30-90$ & 13 & 10 & 35 & 6 & 37 & .3 & 79 \\
\hline 16 & CJ-TW 12 & $08-08-90$ & 26 & 15 & 28 & 49 & 13 & $<.1$ & 63 \\
\hline 17 & CJ-TW 11 & $07-23-90$ & 13 & 9 & 22 & $<1$ & 18 & $<.1$ & 68 \\
\hline 18 & E. Delgado & $08-02-90$ & 39 & 14 & 41 & 20 & 17 & .8 & 47 \\
\hline 19 & Gurabo AAA 6 & $08-20-90$ & 79 & 48 & 88 & 230 & 89 & .3 & 54 \\
\hline 20 & Gurabo AAA 7 & $05-01-90$ & 39 & 30 & 37 & 34 & 48 & .1 & 53 \\
\hline 21 & CJ-TW 17 & $07-10-90$ & 51 & 25 & 23 & 34 & 42 & .2 & 63 \\
\hline 22 & CJ-TW $21 \mathrm{~A}$ & $07-12-90$ & 43 & 13 & 24 & 27 & 30 & $<.1$ & 57 \\
\hline 23 & O. Dávila & $04-26-90$ & 61 & 48 & 65 & 19 & 92 & .3 & 45 \\
\hline 24 & CJ-TW 18 & $07-18-90$ & 63 & 24 & 20 & 23 & 13 & .2 & 47 \\
\hline 25 & P. Morales & $08-23-90$ & 53 & 44 & 53 & 26 & 61 & .3 & 48 \\
\hline 26 & El Bambu AAA & $08-22-90$ & 57 & 19 & 39 & 38 & 36 & .2 & 51 \\
\hline
\end{tabular}


Table 2. Major ions and nutrients in ground water at selected wells and a spring within the Caguas-Juncos alluvial valley, east-central Puerto Rico--Continued

\begin{tabular}{|c|c|c|c|c|c|c|c|c|c|}
\hline $\begin{array}{c}\text { Well } \\
\text { number }\end{array}$ & Well name & $\begin{array}{c}\text { Date of } \\
\text { sampling }\end{array}$ & $\begin{array}{c}\text { Calcium, } \\
\text { dissolved } \\
\text { (mg/L) }\end{array}$ & $\begin{array}{c}\text { Magnesium, } \\
\text { dissolved } \\
\text { (mg/L) }\end{array}$ & $\begin{array}{l}\text { Sodium, } \\
\text { dissolved } \\
\text { (mg/L) }\end{array}$ & $\begin{array}{c}\text { Sulfate, } \\
\text { dissolved } \\
\text { (mg/L) }\end{array}$ & $\begin{array}{c}\text { Chloride, } \\
\text { dissolved } \\
(\mathrm{mg} / \mathrm{L})\end{array}$ & $\begin{array}{c}\text { Flouoride, } \\
\text { dissolved } \\
(\mathrm{mg} / \mathrm{L})\end{array}$ & $\begin{array}{c}\text { Silica, } \\
\text { dissolved } \\
\text { (mg/L as } \\
\mathrm{SiO}_{2}\end{array}$ \\
\hline 27 & Berríos 1 & $04-30-90$ & 80 & 46 & 47 & 59 & 67 & $<.1$ & 38 \\
\hline 28 & Don Sico AAA & $08-22-90$ & 100 & 31 & 48 & 240 & 50 & .2 & 45 \\
\hline 29 & C. Sánchez & $07-09-90$ & 87 & 53 & 91 & 51 & 110 & .3 & 47 \\
\hline 30 & E. Mendoza 1 & $06-28-90$ & 27 & 18 & 33 & 25 & 41 & .1 & 51 \\
\hline 31 & CI-TW 3A & $07-12-90$ & 22 & 13 & 28 & 13 & 39 & .1 & 46 \\
\hline 32 & CJ-TW 4A & $07-16-90$ & 17 & 10 & 21 & 12 & 25 & .1 & 48 \\
\hline 33 & CJ-TW 4B & $07-16-90$ & 41 & 8 & 34 & 13 & 24 & .2 & 37 \\
\hline 34 & Z. Méndez 2 & $08-01-90$ & 50 & 18 & 70 & 110 & 45 & .7 & 32 \\
\hline 35 & Juncos AAA I & $04-23-90$ & 39 & 15 & 30 & 18 & 29 & .1 & 44 \\
\hline 36 & Juncos AAA 2 & $07-31-90$ & 74 & 20 & 50 & 90 & 69 & .2 & 49 \\
\hline 37 & Juncos AAA 7 & $07-31-90$ & 32 & 15 & 23 & 27 & 25 & .5 & 45 \\
\hline 38 & Juncos AAA 5 & $04-23-90$ & 60 & 16 & 45 & 34 & 44 & .1 & 47 \\
\hline 39 & Juncos AAA 3 & $07-30-90$ & 69 & 16 & 45 & 50 & 47 & .1 & 52 \\
\hline 40 & Finca Batey & $08-27-90$ & 16 & 9 & 43 & 30 & 19 & 1.1 & 59 \\
\hline 41 & CJ-TW 16A & $07-10-90$ & 12 & 8 & 20 & 3 & 22 & $<.1$ & 48 \\
\hline 42 & CJ-TW 16B & $07-17-90$ & 12 & 7 & 18 & 11 & 21 & $<.1$ & 36 \\
\hline 43 & CJ-TW 6 & $08-06-90$ & 59 & 25 & 140 & 50 & 160 & .5 & 39 \\
\hline 44 & CJ-TW 23 & $07-17-90$ & 31 & 17 & 99 & 41 & 83 & .4 & 51 \\
\hline 45 & E. Berrios 2 & $08-23-90$ & 35 & 19 & 34 & 21 & 41 & .2 & 50 \\
\hline 46 & Santa Ana 1 & $04-30-90$ & 66 & 39 & 43 & 27 & 57 & .2 & 70 \\
\hline 47 & Hermosura 2 & $07-26-90$ & 26 & 11 & 56 & 20 & 48 & .1 & 64 \\
\hline 48 & Robles Mendoza & $07-26-90$ & 13 & 4 & 9 & 14 & 19 & $<.1$ & 16 \\
\hline 49 & Hacienda Cuco 2 & $04-26-90$ & 65 & 34 & 62 & 59 & 69 & .3 & 52 \\
\hline
\end{tabular}


Table 2. Major ions and nutrients in ground water at selected wells and a spring within the Caguas-Juncos alluvial valley, east-central Puerto Rico--Continued

\begin{tabular}{|c|c|c|c|c|c|c|c|c|c|}
\hline $\begin{array}{c}\text { Well } \\
\text { number }\end{array}$ & Well name & $\begin{array}{c}\text { Date of } \\
\text { sampling }\end{array}$ & $\begin{array}{c}\text { Nitrogen, } \\
\mathrm{NO}_{2}+\mathrm{NO}_{3} \\
\text { dissolved } \\
\text { (mg/L as } \mathrm{N} \text { ) }\end{array}$ & $\begin{array}{l}\text { Phospho- } \\
\text { rous, ortho, } \\
\text { dissolved } \\
\text { (mg/L as P) }\end{array}$ & $\begin{array}{c}\text { Iron, } \\
\text { dissolved } \\
(\mu \mathrm{g} / \mathrm{L})\end{array}$ & $\begin{array}{c}\text { Man- } \\
\text { ganese, } \\
\text { dissolved } \\
(\mu g / L)\end{array}$ & $\begin{array}{l}\text { Dissolved } \\
\text { solids } \\
\text { (mg/L) }\end{array}$ & $\begin{array}{c}\text { Alkalinity } \\
(\mathrm{mg} / \mathrm{L} \text { as } \\
\left.\mathrm{CaCO}_{3}\right)\end{array}$ & $\begin{array}{c}\text { Hardness } \\
\text { total } \\
(\mathrm{mg} / \mathrm{L} \text { as } \\
\left.\mathrm{CaCO}_{3}\right)\end{array}$ \\
\hline 1 & Pozo Viejo AAA & $08-27-90$ & 1.6 & 0.03 & 6 & 3 & 301 & 185 & 190 \\
\hline 2 & CJ-TW 15 & $08-08-90$ & 1.2 & .04 & 20 & 58 & 335 & 205 & 220 \\
\hline 3 & Caguas Sugar & $06-27-90$ & $<.1$ & .01 & 4,400 & 620 & 437 & 197 & 220 \\
\hline 4 & Caguitas Spring & $06-29-90$ & $<1$ & $<.01$ & 47 & 89 & 1,950 & 34 & 1,100 \\
\hline 5 & Quality Elect. & $09-10-90$ & 3.8 & .03 & 9 & 4 & 486 & 238 & 270 \\
\hline 6 & CJ-TW 20 & $07-11-90$ & $<.1$ & .04 & 170 & 800 & 595 & 223 & 150 \\
\hline 7 & CJ-TW 1 & $08-07-90$ & $<.1$ & $<.01$ & 2,520 & 6,200 & 2,650 & 147 & 1,400 \\
\hline 8 & Cartagena 2 & $08-30-90$ & 9.1 & .03 & 5 & $<1$ & 588 & 288 & 430 \\
\hline 10 & CJ-TW 22 & $08-02-90$ & .6 & .06 & 12 & 28 & 310 & 188 & 200 \\
\hline 11 & CJ-TW 7 & $08-09-90$ & .2 & $<.01$ & 78 & 1,100 & 544 & 367 & 300 \\
\hline 12 & CJ-TW 13 & $08-06-90$ & 3.1 & .10 & 19 & 560 & 240 & 138 & 130 \\
\hline 13 & CJ-TW 19A & $07-19-90$ & 5.7 & .11 & 29 & 23 & 319 & 114 & 140 \\
\hline 14 & Gravero Navarro & $04-19-90$ & $<.1$ & $<.01$ & 200 & 78 & 679 & 185 & 330 \\
\hline 15 & R. Diez & $07-30-90$ & 2.3 & .07 & 41 & 12 & 238 & 95 & 74 \\
\hline 16 & CJ-TW 12 & $08-08-90$ & 1.9 & .08 & 45 & 510 & 260 & 107 & 130 \\
\hline 17 & CJ-TW 11 & $07-23-90$ & 3.8 & .06 & 180 & 910 & - & 89 & 70 \\
\hline 18 & E. Delgado & $08-02-90$ & .1 & .01 & 27 & 67 & 310 & 217 & 160 \\
\hline 19 & Gurabo AAA 6 & $08-20-90$ & .1 & .07 & 9 & 130 & 750 & 264 & 400 \\
\hline 20 & Gurabo AAA 7 & $05-01-90$ & .2 & .03 & 10 & 100 & 374 & 220 & 220 \\
\hline 21 & CJ-TW 17 & $07-10-90$ & 1.1 & .08 & 32 & 2,200 & 354 & 183 & 230 \\
\hline 22 & CJ-TW 21A & $07-12-90$ & $<.1$ & .03 & 130 & 300 & 283 & 147 & 160 \\
\hline 23 & O. Dávila & $04-26-90$ & 2.8 & .02 & 180 & 500 & 542 & 348 & 350 \\
\hline 24 & CJ-TW 18 & $07-18-90$ & $<.1$ & .02 & 12 & 750 & 359 & 277 & 260 \\
\hline 25 & P. Morales & $08-23-90$ & .5 & .02 & 6 & 4 & 472 & 310 & 310 \\
\hline 26 & El Bambu AAA & $08-22-90$ & $<.1$ & .01 & 110 & 110 & 374 & 220 & 220 \\
\hline
\end{tabular}


Table 2. Major ions and nutrients in ground water at selected wells and a spring within the Caguas-Juncos alluvial valley, east-central Puerto Rico--Continued

\begin{tabular}{|c|c|c|c|c|c|c|c|c|c|}
\hline $\begin{array}{c}\text { Well } \\
\text { number }\end{array}$ & Well name & $\begin{array}{c}\text { Date of } \\
\text { sampling }\end{array}$ & $\begin{array}{c}\text { Nitrogen, } \\
\mathrm{NO}_{2}+\mathrm{NO}_{3} \\
\text { dissolved } \\
\text { (mg/L as } \mathrm{N})\end{array}$ & $\begin{array}{l}\text { Phospho- } \\
\text { rous, ortho, } \\
\text { dissolved } \\
\text { (mg/L as P) }\end{array}$ & $\begin{array}{l}\text { Iron, } \\
\text { dissolved } \\
(\mu \mathrm{g} / \mathrm{L})\end{array}$ & $\begin{array}{c}\text { Man- } \\
\text { ganese, } \\
\text { dissolved } \\
(\mu \mathrm{g} / \mathrm{L})\end{array}$ & $\begin{array}{l}\text { Dissolved } \\
\text { solids } \\
\text { (mg/L) }\end{array}$ & $\begin{array}{c}\text { Alkalinity } \\
\text { (mg/L as } \\
\mathrm{CaCO}_{3} \text { ) }\end{array}$ & $\begin{array}{c}\text { Hardness } \\
\text { total } \\
(\mathrm{mg} / \mathrm{L} \text { as } \\
\mathrm{CaCO}_{3} \text { ) }\end{array}$ \\
\hline 27 & Berríos 1 & $04-30-90$ & .7 & $<.01$ & 1,200 & 150 & 548 & 349 & 390 \\
\hline 28 & Don Sico AAA & $08-22-90$ & .1 & $<.01$ & 80 & 110 & 623 & 179 & 380 \\
\hline 29 & C. Sánchez & $07-09-90$ & 2.6 & .02 & 12 & 6 & 690 & 415 & 440 \\
\hline 30 & E. Mendoza 1 & $06-28-90$ & 2.8 & .02 & 47 & 2.100 & 273 & 125 & 140 \\
\hline 31 & CJ-TW 3A & $07-12-90$ & 4.5 & .02 & 14 & 250 & 212 & 82 & 110 \\
\hline 32 & CJ-TW 4A & $07-16-90$ & 2.6 & .01 & 5 & 570 & 183 & 81 & 84 \\
\hline 33 & CJ-TW 4B & $07-16-90$ & 1.0 & .02 & 12 & 89 & 255 & 160 & 130 \\
\hline 34 & Z. Méndez 2 & $08-01-90$ & 1.1 & .01 & 4 & 4 & 445 & 195 & 200 \\
\hline 35 & Juncos AAA 1 & $04-23-90$ & .9 & .03 & 12 & 870 & 277 & 162 & 160 \\
\hline 36 & Juncos AAA 2 & $07-31-90$ & .5 & .03 & 120 & 800 & 479 & 202 & 270 \\
\hline 37 & Juncos AAA 7 & $07-31-90$ & .9 & .02 & 7 & 110 & 249 & 134 & 140 \\
\hline 38 & Juncos AAA 5 & $04-23-90$ & .6 & .04 & 88 & 160 & 367 & 198 & 220 \\
\hline 39 & Juncos AAA 3 & $07-30-90$ & .3 & .02 & 10 & 44 & 413 & 220 & 240 \\
\hline 40 & Finca Batey & $08-27-90$ & 2.4 & .08 & 7 & 4 & 252 & 121 & 78 \\
\hline 41 & CJ-TW 16A & $07-10-90$ & 4.4 & .11 & 100 & 130 & 152 & 61 & 65 \\
\hline 42 & CJ-TW 16B & $07-17-90$ & 3.2 & $<.01$ & 17 & 15 & 138 & 52 & 60 \\
\hline 43 & CJ-TW 6 & $08-06-90$ & .8 & .03 & 40 & 480 & 653 & 294 & 250 \\
\hline 44 & CJ-TW 23 & $07-17-90$ & $<.1$ & .03 & 130 & 870 & 458 & 223 & 150 \\
\hline 45 & E. Berríos 2 & $08-23-90$ & 4.3 & .02 & $<3$ & $<1$ & 285 & 139 & 170 \\
\hline 46 & Santa Ana 1 & $04-30-90$ & 1.1 & .04 & 5 & 2 & 488 & 308 & 330 \\
\hline 47 & Hermosura 2 & $07-26-90$ & 2.3 & .12 & 7 & 8 & 316 & 149 & 110 \\
\hline 48 & Robles Mendoza & $07-26-90$ & .4 & .03 & 40 & 11 & 91 & 25 & 49 \\
\hline 49 & Hacienda Cuco 2 & $04-26-90$ & 2.4 & .05 & 4 & 13 & 512 & 282 & 300 \\
\hline
\end{tabular}


Table 3. Trace metals in ground water at selected wells and a spring within the Caguas-Juncos alluvial valley, east-central Puerto Rico

$[\mu \mathrm{g} / \mathrm{L}$, micrograms per liter; $n d$, not detected]

\begin{tabular}{|c|c|c|c|c|c|c|c|c|c|c|c|}
\hline $\begin{array}{l}\text { Well } \\
\text { number }\end{array}$ & Well name & $\begin{array}{l}\text { Date of } \\
\text { sampling }\end{array}$ & $\begin{array}{l}\text { Barium, } \\
\text { dis- } \\
\text { solved } \\
(\mu \mathrm{g} / \mathrm{L})\end{array}$ & $\begin{array}{c}\text { Cadmium } \\
\text { dissolved } \\
(\mu \mathrm{g} / \mathrm{L})\end{array}$ & $\begin{array}{l}\text { Lead, } \\
\text { dis- } \\
\text { solved } \\
(\mu g / L)\end{array}$ & $\begin{array}{c}\text { Molyb- } \\
\text { denum, } \\
\text { dis- } \\
\text { solved } \\
(\mu \mathrm{g} / \mathrm{L})\end{array}$ & $\begin{array}{l}\text { Silver, } \\
\text { dis- } \\
\text { solved } \\
(\mu \mathrm{g} / \mathrm{L})\end{array}$ & $\begin{array}{l}\text { Stron- } \\
\text { tium, } \\
\text { dis- } \\
\text { solved } \\
(\mu \mathrm{g} / \mathrm{L})\end{array}$ & $\begin{array}{l}\text { Vana- } \\
\text { dium, } \\
\text { dis- } \\
\text { solved } \\
(\mu g / L)\end{array}$ & $\begin{array}{l}\text { Zinc, } \\
\text { dis- } \\
\text { solved } \\
(\mu \mathrm{g} / \mathrm{L})\end{array}$ & $\begin{array}{c}\text { Lithium, } \\
\text { dis- } \\
\text { solved } \\
(\mu \mathrm{g} / \mathrm{L})\end{array}$ \\
\hline 4 & Caguitas Spring & $06-29-90$ & 15 & nd & nd & nd & 5 & 1,800 & nd & nd & 70 \\
\hline 5 & Quality Elect. & $09-10-90$ & 11 & nd & nd & nd & nd & 280 & 27 & 13 & nd \\
\hline 7 & CJ-TW 1 & $08-07-90$ & 61 & nd & nd & nd & nd & 2,000 & nd & 36 & 63 \\
\hline 14 & Gravero Navarro & $04-19-90$ & 8 & nd & nd & nd & 1 & 580 & nd & 63 & 33 \\
\hline 19 & Gurabo AAA 6 & $08-20-90$ & 9 & 1 & 10 & nd & nd & 220 & 9 & 8 & nd \\
\hline 20 & Gurabo AAA 7 & $05-01-90$ & 24 & nd & nd & nd & nd & 520 & 16 & 14 & 6 \\
\hline 32 & CJ-TW 4A & $07-16-90$ & 170 & 1 & 10 & nd & nd & 120 & nd & 16 & nd \\
\hline 33 & CJ-TW 4B & $07-16-90$ & 60 & nd & nd & nd & nd & 220 & 7 & 5 & nd \\
\hline 35 & Juncos AAA 1 & $04-23-90$ & 140 & nd & nd & nd & nd & 230 & nd & 14 & 5 \\
\hline 36 & Juncos AAA 2 & $07-31-90$ & 160 & 1 & nd & nd & I & 410 & nd & 6 & 10 \\
\hline 37 & Juncos AAA 7 & $07-31-90$ & 54 & nd & nd & nd & 1 & 190 & nd & 8 & nd \\
\hline 38 & Juncos AAA 5 & $04-23-90$ & 81 & nd & nd & nd & nd & 280 & nd & 4 & 5 \\
\hline 39 & Juncos AAA 3 & $07-30-90$ & 22 & nd & nd & nd & nd & 300 & 9 & 22 & 8 \\
\hline 40 & Finca Batey & $08-27-90$ & 84 & nd & nd & nd & 1 & 140 & nd & 120 & nd \\
\hline 41 & CJ-TW 16A & $07-10-90$ & 290 & 1 & nd & nd & nd & 92 & nd & 8 & nd \\
\hline 42 & CJ-TW 16B & $07-17-90$ & 260 & nd & nd & nd & nd & 61 & nd & 9 & nd \\
\hline 43 & CJ-TW 6 & $08-06-90$ & 220 & nd & nd & nd & nd & 400 & nd & 15 & 6 \\
\hline 44 & CJ-TW 23 & $07-17-90$ & 200 & nd & nd & nd & nd & 290 & nd & 11 & nd \\
\hline 46 & Santa Ana 1 & $04-30-90$ & 3 & 2 & 20 & 10 & 2 & 240 & 60 & 16 & 10 \\
\hline
\end{tabular}


Table 4. Volatile synthetic organic chemicals in ground water at selected wells within the Caguas-Juncos alluvial valley, east-central Puerto Rico

[mg/L, micrograms; nd, not detected]

\begin{tabular}{|c|c|c|c|c|c|c|c|c|c|c|}
\hline $\begin{array}{l}\text { Well } \\
\text { number }\end{array}$ & Well name & $\begin{array}{l}\text { Date of } \\
\text { sampling }\end{array}$ & $\begin{array}{l}\text { Chloro- } \\
\text { form, } \\
\text { total } \\
(\mu \mathrm{g} / \mathrm{L})\end{array}$ & $\begin{array}{c}\text { Dichloro- } \\
\text { difluoro- } \\
\text { methane, } \\
\text { total } \\
(\mu g / L)\end{array}$ & $\begin{array}{c}\text { Dichloro- } \\
\text { ethylene, } \\
\text { total } \\
(\mu \mathrm{g} / \mathrm{L})\end{array}$ & $\begin{array}{c}\text { Trichloro- } \\
\text { ethylene, } \\
\text { total } \\
(\mu \mathrm{g} / \mathrm{L})\end{array}$ & $\begin{array}{c}\text { Benzene, } \\
\text { total } \\
(\mu \mathrm{g} / \mathrm{L})\end{array}$ & $\begin{array}{c}\text { Ethyl- } \\
\text { benzene, } \\
\text { total } \\
(\mu \mathrm{g} / \mathrm{L})\end{array}$ & $\begin{array}{c}\text { Toluene, } \\
\text { total } \\
(\mu \mathrm{g} / \mathrm{L})\end{array}$ & $\begin{array}{c}\text { Xylene, } \\
\text { total } \\
(\mu \mathrm{g} / \mathrm{L})\end{array}$ \\
\hline 1 & Pozo Viejo AAA & $08-27-90$ & nd & nd & nd & nd & nd & nd & nd & nd \\
\hline 5 & Quality Elect. & $09-10-90$ & 2.0 & nd & 0.4 & 0.7 & nd & nd & nd & nd \\
\hline 7 & CJ-TW 1 & $08-07-90$ & nd & nd & nd & nd & nd & nd & nd & nd \\
\hline 9 & CJ-TW2 & $09-11-90$ & nd & nd & nd & nd & 5,800 & 1,200 & 12.000 & 15,000 \\
\hline 15 & R. Diez & $07-30-90$ & 2.5 & nd & nd & nd & nd & nd & nd & nd \\
\hline 17 & CJ-TW 11 & $07-23-90$ & nd & nd & nd & nd & nd & nd & nd & nd \\
\hline 19 & Gurabo AAA 6 & $05-01-90$ & nd & nd & nd & nd & nd & nd & nd & nd \\
\hline 20 & Gurabo AAA 7 & $08-20-90$ & nd & nd & nd & nd & nd & nd & nd & nd \\
\hline 29 & C. Sánchez & $07-09-90$ & 0.9 & nd & nd & nd & nd & nd & nd & nd \\
\hline 32 & CJ-TW 4A & $07-16-90$ & nd & nd & nd & nd & nd & nd & nd & nd \\
\hline 35 & Juncos AAA 1 & $04-23-90$ & nd & nd & nd & nd & nd & nd & nd & nd \\
\hline 36 & Juncos AAA 2 & $07-31-90$ & nd & nd & nd & nd & nd & nd & nd & nd \\
\hline 37 & Juncos AAA 7 & $07-30-90$ & nd & nd & nd & nd & nd & nd & nd & nd \\
\hline 38 & Juncos AAA 5 & $04-23-90$ & nd & nd & nd & nd & nd & nd & nd & nd \\
\hline 40 & Finca Batey & $08-27-90$ & nd & nd & nd & nd & nd & nd & nd & nd \\
\hline 41 & CJ-TW 16A & $07-10-90$ & nd & nd & nd & nd & nd & nd & nd & nd \\
\hline 43 & CJ-TW 6 & $08-06-90$ & nd & nd & nd & nd & nd & nd & nd & nd \\
\hline 44 & CJ-TW 23 & $07-17-90$ & nd & nd & nd & nd & nd & nd & nd & nd \\
\hline 46 & Santa Ana 1 & $04-30-90$ & 0.4 & 3.6 & nd & nd & nd & nd & nd & nd \\
\hline
\end{tabular}


Table 5. Water-quality regulations applicable to drinking water and potable water sources in Puerto Rico [ $\mu \mathrm{g} / \mathrm{L}$, micrograms per liter; mg/L, milligrams per liter; --, regulation not established]

\begin{tabular}{|c|c|c|c|c|}
\hline \multirow[b]{2}{*}{ Chemical } & \multicolumn{2}{|c|}{ U.S. Environmental Protection Agency (1994) } & \multirow{2}{*}{$\begin{array}{c}\begin{array}{c}\text { Puerto Rico Department } \\
\text { of Health (1989) }\end{array} \\
\begin{array}{c}\text { Drinking-Water } \\
\text { Maximum Contaminant } \\
\text { Level (PRMCL) }\end{array}\end{array}$} & \multirow{2}{*}{$\begin{array}{c}\text { Puerto Rico } \\
\text { Environmental Quality } \\
\text { Board (1990) }\end{array}$} \\
\hline & $\begin{array}{c}\text { Drinking-water } \\
\text { Maximum Contaminant } \\
\text { Level }(\mathrm{MCL})^{1}\end{array}$ & $\begin{array}{c}\text { Drinking-Water } \\
\text { Secondary Maximum } \\
\text { Contaminant Level } \\
(\mathrm{SMCL})^{2}\end{array}$ & & \\
\hline \multicolumn{5}{|l|}{ Inorganics } \\
\hline Sulfate $(\mathrm{mg} / \mathrm{L})$ & -- & 250 & -- & -- \\
\hline Chloride (mg/L) & -- & 250 & -- & -- \\
\hline Fluoride (mg/L) & 4 & 2 & - & -- \\
\hline Iron $(\mu \mathrm{g} / \mathrm{L})$ & -- & 300 & -- & -- \\
\hline Manganese $(\mu \mathrm{g} / \mathrm{L})$ & -- & 50 & -- & -- \\
\hline Dissolved solids (mg/L) & -- & 500 & - & -- \\
\hline Barium $(\mu \mathrm{g} / \mathrm{L})$ & 2,000 & -- & 1,000 & - \\
\hline Cadmium $(\mu \mathrm{g} / \mathrm{L})$ & 5 & -- & 10 & 5 \\
\hline Lead $(\mu \mathrm{g} / \mathrm{L})$ & -- & -- & 50 & 50 \\
\hline Silver $(\mu \mathrm{g} / \mathrm{L})$ & -- & 100 & 50 & -- \\
\hline Zinc $(\mu \mathrm{g} / \mathrm{L})$ & -- & 5,000 & -- & - \\
\hline \multicolumn{5}{|l|}{ Organics } \\
\hline Dichloroethylene $(\mu \mathrm{g} / \mathrm{L})$ & 7 & -- & 7 & 7 \\
\hline Trichloroethylene $(\mu \mathrm{g} / \mathrm{L})$ & 5 & -- & 5 & 5 \\
\hline Benzene $(\mu \mathrm{g} / \mathrm{L})$ & 5 & - & 5 & 5 \\
\hline Ethylbenzene $(\mu \mathrm{g} / \mathrm{L})$ & 700 & - & - & -- \\
\hline Tolune $(\mu \mathrm{g} / \mathrm{L})$ & 1,000 & -- & - & - \\
\hline Xylene $(\mu \mathrm{g} / \mathrm{L})$ & 10,000 & -- & -- & -- \\
\hline
\end{tabular}

${ }^{1}$ Maximum contaminant level (MCL) - Enforceable, health based regulation.

${ }^{2}$ Secondary maximum contaminant level (SMCL) - Not enforceable, aesthically based regulation. At high concentrations health implications as well as aesthetic degradation may also exist. SMCL are not Federally enforceable but are intended as guidelines for the States.

${ }^{3}$ Maximum permissible concentration (MPC) - Enforceable, health based regulation for protection of water bodies used as potable water sources. 


\section{Ground-Water Quality in the Caguas-Juncos Valley, Puerto Rico, April to October 1990}

By José M. Rodríguez and Juan C. Puig

U.S. GEOLOGICAL SURVEY

Open-File Report 96-139

Prepared in cooperation with the

PUERTO RICO AQUEDUCT AND SEWER AUTHORITY 


\section{REFERENCES}

Brown, Edward, Skougstad, M.W., and Fishman, M.J., 1970, Methods for the collection and analysis of water samples for dissolved minerals and gases: U.S Geological Survey Techniques of Water Resources Investigations, Book 5, Chap. A1, 160 p.

Claassen, H.C., 1982, Guidelines and techniques for obtaining water samples that accurately represent the water chemistry of an aquifer: U.S. Geological Survey Open-File Report 82-1024, $24 \mathrm{p}$.

Hem, J.D., 1989, Study and interpretation of the chemical characteristics of natural water (3rd ed.): U.S. Geological Survey Water-Supply Paper 2254, 263 p.

Puerto Rico Department of Health, 1989, Summary of primary drinking water regulations: Puerto Rico Department of Health, Regulation No. 50, Water Supply Supervision Program, 9 p.

Puerto Rico Environmental Quality Board, 1990, Water quality standards regulation of Puerto Rico: Puerto Rico Environmental Quality Board R-90-26-1, 104 p.
Puig, J.C., and Rodríguez, J.M., 1993, Ground-water resources of the Caguas-Juncos Valley, Puerto Rico: U.S. Geological Survey Water-Resources Investigations Report 91-4079, $52 \mathrm{p}$.

U.S. Department of Commerce, 1991, 1990 Census of population and housing - Summary population and housing characteristics, Puerto Rico: U.S. Department of Commerce, Bureau of Census, CPH-1-53,199 p., 7 appendices.

1982, 1980 Census of population and housing, Puerto Rico: U.S. Department of Commerce, Bureau of Census, PHC80-V-53, p. 53-1 to 53-11.

U.S. Environmental Protection Agency, 1994, National primary drinking-water standards: U.S. Environmental Protection Agency, Office of Drinking Water, EPA 810-F-94001A, variously paged.

Wood, W.W., 1976, Guidelines for collection and field analysis of ground-water samples for selected unstable constituents: U.S. Geological Survey Techniques of Water-Resources Investigations, Book 1, Chap. D2, 24 p. 



\title{
U.S. DEPARTMENT OF THE INTERIOR BRUCE BABBITT, Secretary
}

\author{
U.S. GEOLOGICAL SURVEY
}

Gordon P. Eaton, Director

For additional information write to:

Copies of this report can be purchased from:

District Chief

U.S. Geological Survey

GSA Center, Suite 400-15

651 Federal Drive

San Juan, Puerto Rico 00965
U.S. Geological Survey

Earth Science Information Center

Open-File Reports Section, MS 517

Box 25286, Denver Federal Center

Denver, CO 80225 


\section{CONTENTS}

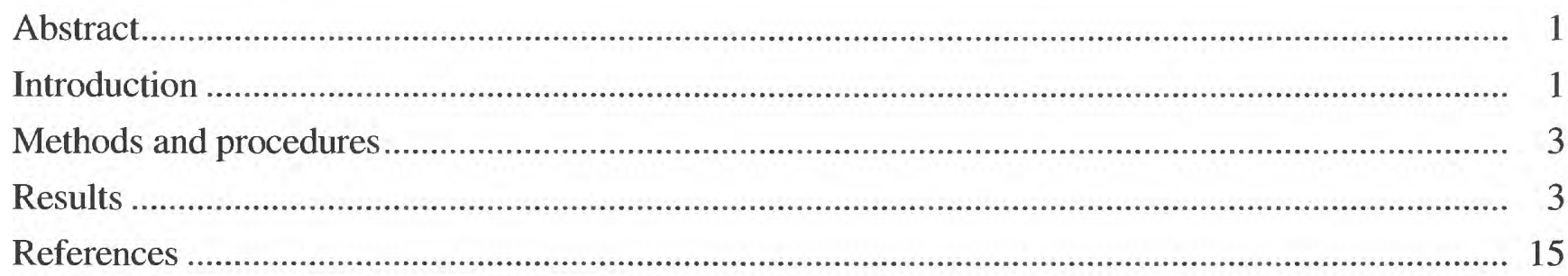

FIGURES

1. Map showing location of the Caguas-Juncos alluvial valley in east-central Puerto Rico

2. Map showing location of sample-collection wells and spring in the Caguas-Juncos alluvial valley, east-central Puerto Rico

\section{TABLES}

1. Information and ground-water field measurements from selected wells and a spring within the Caguas-Juncos alluvial valley, east-central Puerto Rico

2. Major ions and nutrients in ground water at selected wells and a spring within the Caguas-Juncos alluvial valley, east-central Puerto Rico

3. Trace metals in ground water at selected wells and a spring within the Caguas-Juncos alluvial valley, east-central Puerto Rico

4. Volatile synthetic organic chemicals in ground water at selected wells within the Caguas-Juncos alluvial valley, east-central Puerto Rico

5. Water-quality regulations applicable to drinking water and potable water sources in Puerto Rico 
CONVERSION FACTORS, ABBREVIATED WATER-QUALITY UNITS, AND ACRONYMS

\begin{tabular}{ccc}
\hline Multiply & By & To obtain \\
\hline million gallons per day & Flow & \\
& 0.04381 & cubic meter per second \\
& Length & \\
foot & 0.3048 & meter \\
Temperature in degrees Celsius $\left({ }^{\circ} \mathrm{C}\right)$ may be converted to degrees Fahrenheit $\left({ }^{\circ} \mathrm{F}\right)$ as follows \\
\end{tabular}

Abbreviated water-quality units and terms used in report:

micrograms per liter $(\mu \mathrm{g} / \mathrm{L})$

milligrams per liter $(\mathrm{mg} / \mathrm{L})$

microsiemens per centimeter at $25^{\circ} \mathrm{C}(\mu \mathrm{S} / \mathrm{cm})$

Acronyms used in report:

Maximum contaminant limit (MCL)

Maximum permissible concentration (MPC)

Puerto Rico Aqueduct and Sewer Authority (PRASA)

Secondary maximum contaminant level (SMCL)

U.S. Geological Survey (USGS)

Volatile synthetic organic chemicals (VOC) 


\title{
Ground-Water Quality in the Caguas-Juncos Valley, Puerto Rico, April to October 1990
}

\author{
By José M. Rodríguez and Juan C. Puig
}

\section{ABSTRACT}

Ground water from 48 wells and 1 spring in the Caguas-Juncos Valley was sampled and analyzed for major ions, nutrients, trace metals, and volatile synthetic organic chemicals from April to October 1990. This report presents the results of physical and chemical analyses made on these water samples.

Dissolved-solids concentrations exceeded 500 milligrams per liter in 28 percent of the samples. Concentrations of trace metals were below the maximum contaminant levels set by Federal and Commonwealth agencies. Volatile synthetic organic chemicals were detected in water from five wells, four of which were located in urban and industrial areas. Concentrations of benzene, ethylbenzene, toluene, and xylene detected in samples from one observation well exceeded the maximum contaminant levels set be Federal and Commonwealth agencies.

\section{INTRODUCTION}

The Caguas-Juncos Valley is an interior alluvial valley located within the Río Grande de Loíza drainage basin in east-central Puerto Rico (fig. 1). The valley is divided into two subareas: the Caguas Valley and the Gurabo-Juncos Valley. The population growth (from 167,000 in 1980 to 192,000 in 1990) in the municipalities of Caguas, Gurabo, and Juncos, located in the Caguas-Juncos Valley, has created an increased demand for potable water (U.S. Department of Commerce, 1982 and 1991). The public-water supply in the Caguas-Juncos Valley was 21 million gallons per day during 1986, of which 16 percent was derived from ground water (Puig and Rodríguez, 1993).

As part of a study to evaluate the potential for additional development of ground water in the Caguas-Juncos Valley, the U.S. Geological Survey (USGS), in cooperation with the Puerto Rico Aqueduct and Sewer Authority (PRASA) conducted a reconnaissance of ground-water quality from April to October 1990. A previous study conducted by the USGS on the groundwater quality at selected public-water supply wells throughout Puerto Rico (Senén GuzmánRíos, USGS, written commun., 1989) detected the presence of volatile synthetic organic chemicals (VOC) in ground water from a well located near Caguas. This report presents the results of a synoptic survey of the ground-water quality in the Caguas-Juncos Valley. 


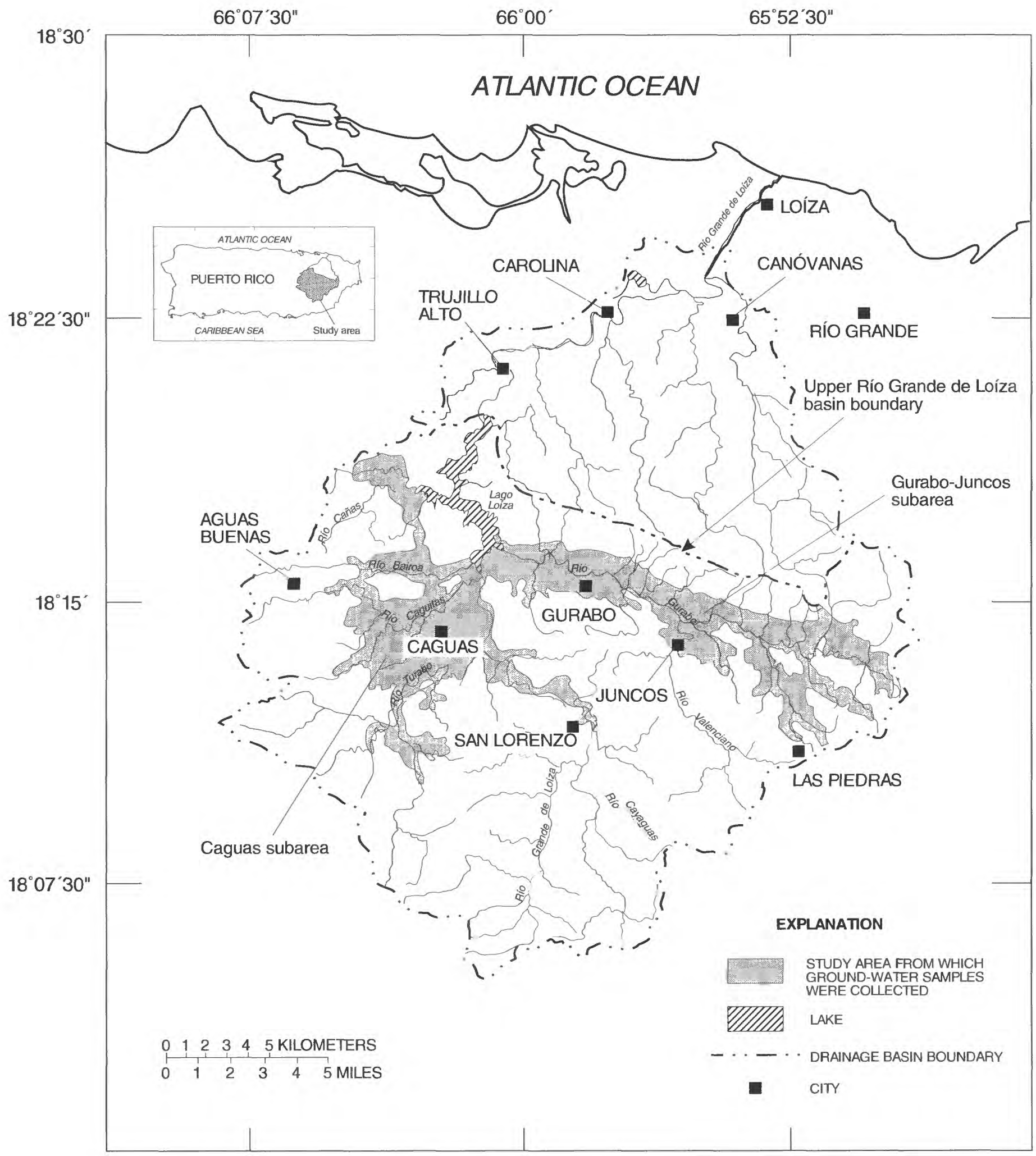

Figure 1. Location of the Caguas-Juncos alluvial valley in east-central Puerto Rico. 


\section{METHODS AND PROCEDURES}

Ground-water samples were collected from 48 wells and 1 spring. Sampling techniques described by Brown and others (1970), Claassen (1982), and Wood (1976) were followed.

Eighteen sampling sites were located in the Caguas Valley and 31 in the Gurabo-Juncos Valley (fig. 2). Ground-water samples from production wells were collected prior to chlorination and as close as possible to the well head. Samples from observation wells were collected after three times the volume of water in the well casing had been removed with a submersible pump. The same procedure was used to collect samples for VOC analysis, except that the pump was removed from the observation well and the water level was allowed to recover before the sample was collected. Samples for VOC analyses were collected with a bailer made of ${ }^{1}$ Teflon ${ }^{\mathrm{TM}}$ which was lowered to the midpoint of the screened interval in the well.

Field measurements were made for temperature, specific conductance, $\mathrm{pH}$, and alkalinity for each ground-water sample. Samples were processed and preserved in the field with standard USGS water-quality field techniques and sent to the USGS National Water-Quality Laboratory at Arvada, Colorado, for analysis. All samples (except samples from well 9) were analyzed for major ions and nutrients. Water samples collected from public-supply wells and wells located near landfills (active or inactive) and industrial sites, were also analyzed for trace metals, VOC, or both.

\footnotetext{
${ }^{1}$ Any use of trade, product, or firm names is for descriptive purposes only and does not implies endorsement by the U.S. Government.
}

\section{RESULTS}

Information on well location and construction, water use, and field measurements for temperature, specific conductance, $\mathrm{pH}$, and alkalinity for all sample-collection wells in the study area is presented in table 1 . Results of the laboratory analyses for major ions, trace metals, and VOC are summarized in tables 2 through 4. Water-quality data obtained from this study (tables 2 to 4) are compared with water-quality standards established by the Puerto Rico Environmental Board (1990), the Puerto Rico Department of Health (1989), and the U.S. Environmental Protection Agency (1994).

Calcium concentrations in ground-water samples collected from wells in the CaguasJuncos alluvial valley ranged from 12 to 480 milligrams per liter (mg/L). Samples from wells in the Caguas subarea had the highest concentration of calcium. Sodium, chloride, sulfate, and dissolved-solids concentrations ranged from 9 to $280 \mathrm{mg} / \mathrm{L} ; 13$ to $180 \mathrm{mg} / \mathrm{L}$; less than 1 to $1,600 \mathrm{mg} / \mathrm{L}$; and 91 to $2,650 \mathrm{mg} / \mathrm{L}$, respectively. Iron concentrations ranged from less than 3 to $4,400 \mu \mathrm{g} / \mathrm{L}$ and manganese concentrations from less than 1 to $6,200 \mu \mathrm{g} / \mathrm{L}$.

Sulfate concentrations in water samples from well 7 and Caguitas Spring (site 4; table 2) exceeded the secondary drinking-water maximum contaminant level (SMCL) (250 mg/L; table 5). Dissolved-solids concentrations exceeded the SMCL ( $500 \mathrm{mg} / \mathrm{L}$ ) in 13 (28 percent) of 47 samples (table 2). Iron concentrations in water from wells 3, 7, and 27 (table 2) exceeded the SMCL $(300 \mu \mathrm{g} / \mathrm{L})$. Manganese concentrations exceeded the SMCL $(50 \mu \mathrm{g} / \mathrm{L})$ in 31 of 48 samples. Values for hardness exceeded $180 \mathrm{mg} / \mathrm{L}$ in 27 of 48 samples. Water with hardness values above $180 \mathrm{mg} / \mathrm{L}$ is classified as "very hard" (Hem, 1989). 


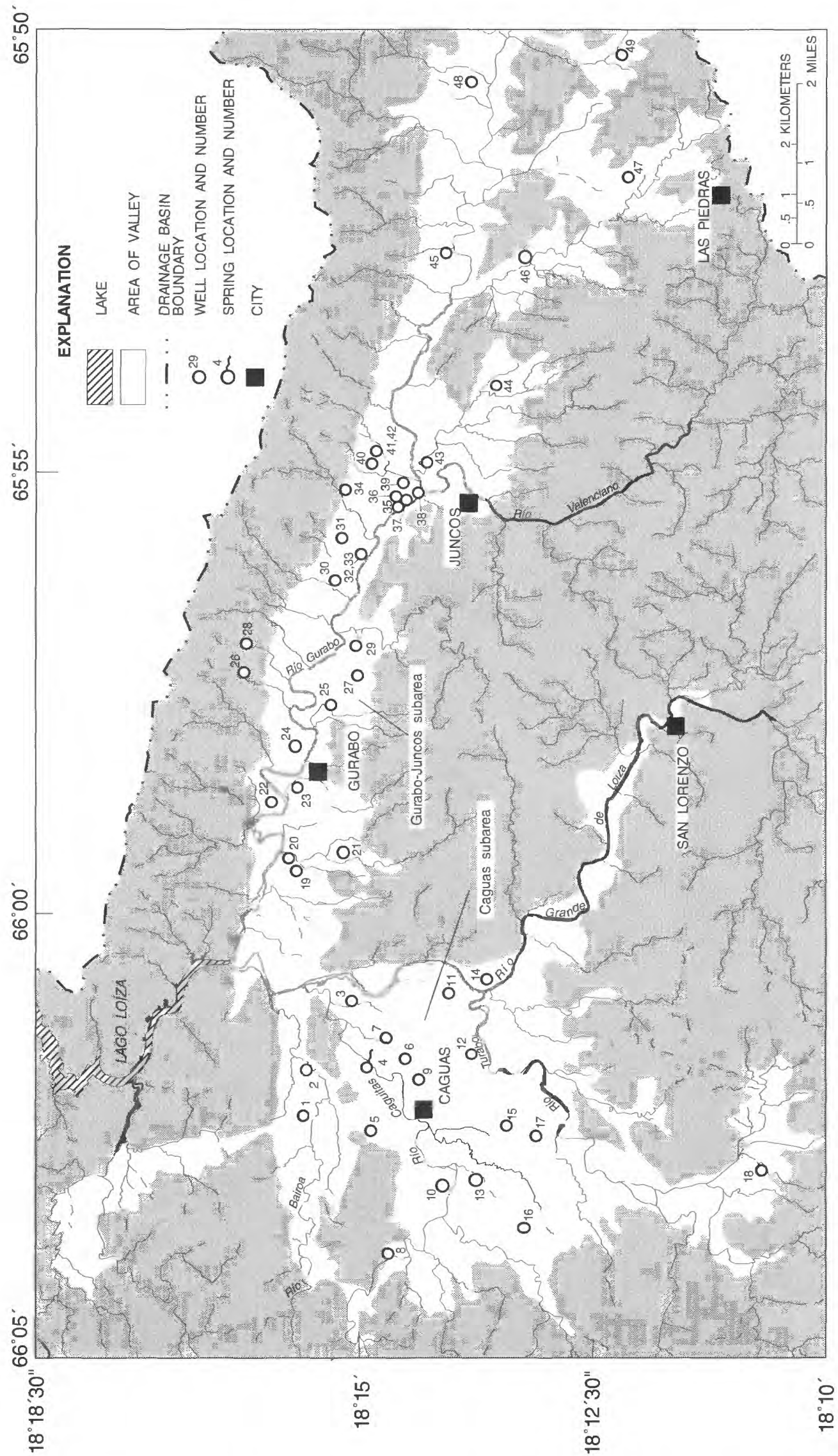

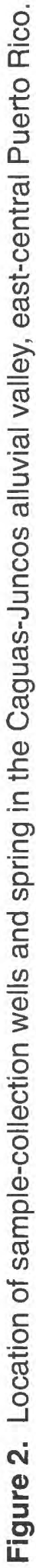

